\title{
Halogenated Ether, Alcohol, and Alkane Anesthetics Activate TASK-3 Tandem Pore Potassium Channels Likely through a Common Mechanism ${ }^{\mathrm{s}}$
}

\author{
Anita Luethy, James D. Boghosian, Rithu Srikantha, and Joseph F. Cotten
}

Department of Anesthesia, Critical Care, and Pain Medicine, Massachusetts General Hospital, Boston, Massachusetts (A.L., J.D.B., and J.F.C.); Department of Anesthesia, Kantonsspital Aarau, Aarau, Switzerland (A.L.); Carver College of Medicine, University of lowa, lowa City, lowa (R.S.)

Received January 7, 2017; accepted March 20, 2017

\section{ABSTRACT}

The TWIK-related acid-sensitive potassium channel 3 (TASK-3; KCNK9) tandem pore potassium channel function is activated by halogenated anesthetics through binding at a putative anestheticbinding cavity. To understand the pharmacologic requirements for TASK-3 activation, we studied the concentration-response of TASK-3 to several anesthetics (isoflurane, desflurane, sevoflurane, halothane, $\alpha$-chloralose, 2,2,2-trichloroethanol [TCE], and chloral hydrate), to ethanol, and to a panel of halogenated methanes and alcohols. We used mutagenesis to probe the anesthetic-binding cavity as observed in a TASK-3 homology model. TASK-3 activation was quantified by Ussing chamber voltage clamp analysis. We mutagenized the residue Val-136, which lines the anesthetic-binding cavity, its flanking residues (132 to 140), and Leu-122, a pore-gating residue. The 2-halogenated ethanols activate wild-type TASK-3 with the following rank order efficacy (normalized current [95\% confidence interval]): 2,2,2-tribromo(267\% [240-294]) > 2,2,2-trichloro-(215\% [196-234]) > chloral hydrate $(165 \%$ [161-176]) $>2,2$-dichloro- $>2$-chloro $\approx 2,2,2$ trifluoroethanol $>$ ethanol. Similarly, carbon tetrabromide $(296 \%$ [245-346]), carbon tetrachloride (180\% [163-196]), and 1,1,1,3,3,3hexafluoropropanol (200\% [194-206]) activate TASK-3, whereas the larger carbon tetraiodide and $\alpha$-chloralose inhibit. Clinical agents activate TASK-3 with the following rank order efficacy: halothane (207\% [202-212]) > isoflurane (169\% [161-176]) $>$ sevoflurane (164\% [150-177]) > desflurane (119\% [109-129]). Mutations at and near residue-136 modify TCE activation of TASK3, and interestingly M159W, V136E, and L122D were resistant to both isoflurane and TCE activation. TASK-3 function is activated by a multiple agents and requires a halogenated substituent between $\sim 30$ and $232 \mathrm{~cm}^{3} / \mathrm{mol}$ volume with potency increased by halogen polarizeability. Val-136 and adjacent residues may mediate anesthetic binding and stabilize an open state regulated by pore residue Leu-122. Isoflurane and TCE likely share commonalities in their mechanism of TASK-3 activation.

\section{Introduction}

The TWIK-related acid-sensitive potassium channel 3 (TASK-3; KCNK9) protein is a member of the tandem pore $\left(\mathrm{K}_{2 \mathrm{p}}\right)$ potassium channel family, a collection of 15 channels that regulate neuronal excitability (Yost, 2003). TASK-3 function is inhibited by extracellular acidic $\mathrm{pH}$ and by activation of $\mathrm{G} \alpha_{\mathrm{Q} / 11}$-coupled G protein coupled receptors (GPCRs), which are involved in central nervous system arousal and consciousness (e.g., orexin, muscarinic, adrenergic, and histamine receptors) (Talley and Bayliss, 2002; Franks, 2008). TASK-3 and other tandem pore channel functions are activated by halogenated volatile anesthetics, which causes neuronal hyperpolarization

This research was supported the Massachusetts General Hospital Department of Anesthesia, Critical Care \& Pain Medicine; the National Institutes of Health National Heart Lung and Blood Institute [Grant R01HL117871]; and Kantonsspital Aarau, Aarau, Switzerland (1410.000.062).

https://doi.org/10.1124/mol.117.108290.

S This article has supplemental material available at molpharm. aspetjournals.org. and quiescence (Franks and Lieb, 1988; Patel et al., 1999; Conway and Cotten, 2012); therefore, tandem pore channels may mediate some aspects of anesthesia. Importantly, knockout mice lacking TASK-3 channels or TREK-1 channels, also a tandem pore member, are resistant to halothane- and isofluraneinduced loss of righting and immobility (Heurteaux et al., 2004; Pang et al., 2009; Lazarenko et al., 2010).

The mechanisms of TASK-3 activation by anesthetics and the drug structural requirements necessary to activate TASK3 are minimally understood. TASK-3 activation studies have been limited to several clinical anesthetics: TASK-3 is activated by isoflurane, halothane, and chloroform; conversely, TASK-3 is unaffected by nonhalogenated nitrous oxide, cyclopropane, propofol, and xenon and is inhibited by local anesthetics (e.g., bupivicaine) (Kindler et al., 1999; Talley and Bayliss, 2002; Gruss et al., 2004; Andres-Enguix et al., 2007). TCE (2,2,2tricholoroethanol), an active metabolite of the hypnotic chloral hydrate, a $\mathrm{GABA}_{\mathrm{A}}$ chloride channel activator, activates TREK-1 and TRAAK tandem pore potassium channels (Krasowski et al.,

ABBREVIATIONS: DMSO, dimethylsulfoxide; FRT, Fischer rat thyroid; GPCR, G protein coupled receptor; HFP, 1,1,1,3,3,3-hexafluoropropanol; LyTASK, Lymnaea TASK; m3 GPCR, m3 muscarinic G protein coupled receptor; NEM, $N$-ethylmaleimide; PCR, polymerase chain reaction; TASK, TWIK-related acid-sensitive potassium channel; TASK-3/TREK-1, 243-VLRFLT-248, > 243-GDWLRV-249; TBE, 2,2,2-tribromoethanol; TCE, 2,2,2tricholoroethanol. 
1998; Krasowski and Harrison, 2000; Harinath and Sikdar, 2004; Parelkar et al., 2010). The effects of TCE on TASK-3 have not been reported.

Molecular chimera and mutagenesis studies have identified TASK-3 mutants that are resistant to activation by halogenated anesthetics. Mutants involve residues Met-159 and 243-VLRFLT-248 (aka "TASK-3/TREK-1" mutant) in the intracellular portions of transmembrane domains 3 and 4, respectively (Fig. 1) (Patel et al., 1999; Talley and Bayliss, 2002; Andres-Enguix et al., 2007). Notably, covalent modification of a cysteine residue introduced at TASK-3 residue-159 (M159C) causes channel activation, suggesting perturbations in this "pocket" activate TASK-3 (Conway and Cotten, 2012). Tandem pore channel crystal structures for TWIK-1, TRAAK, and TREK-2 have been solved and have enabled homology model construction (Brohawn et al., 2012; Miller and Long, 2012; Lolicato et al., 2014; Dong et al., 2015). In a Lymnaea TASK

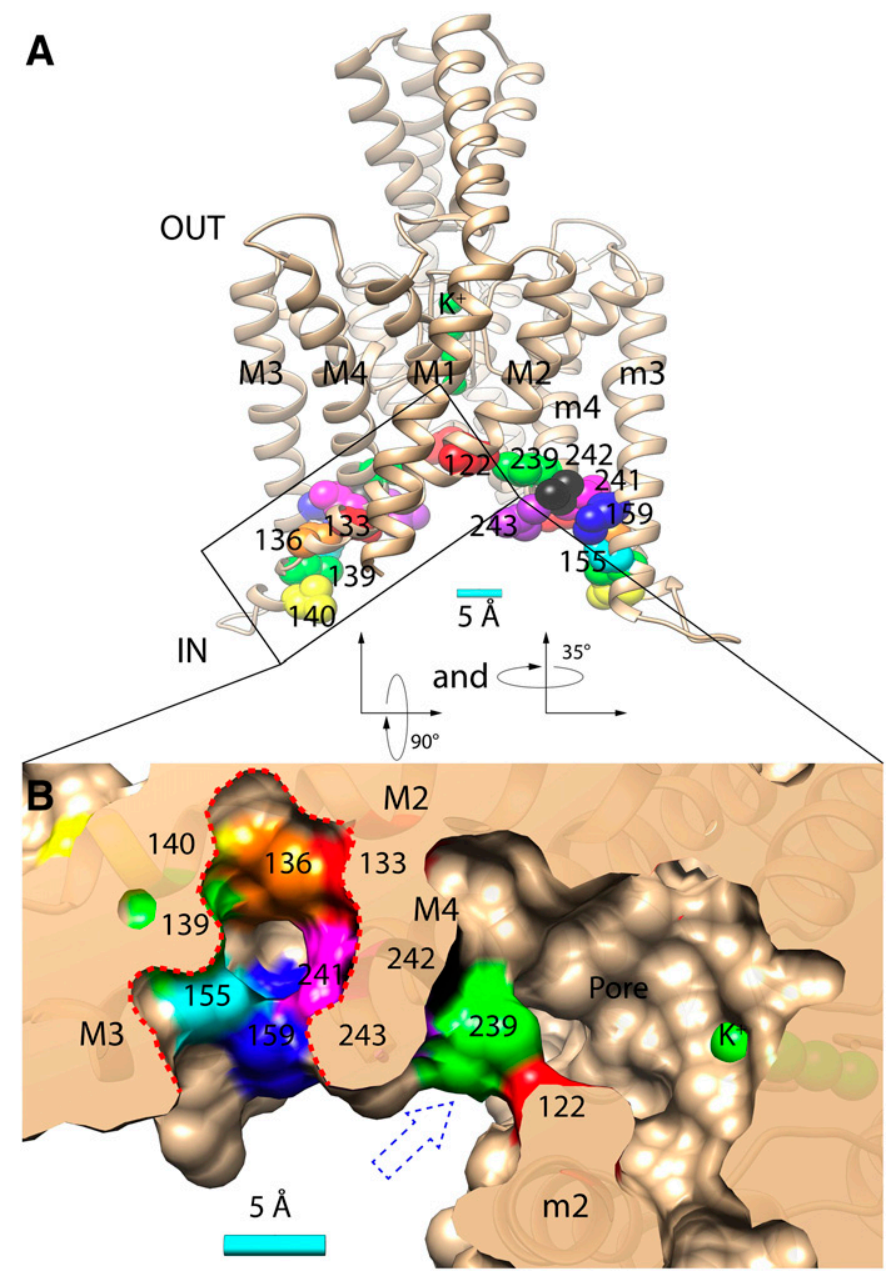

Fig. 1. (A) Homology model of TASK-3 potassium channel (spanning residues Gln-4 to Val-243) with relevant residues indicated: Leu-122 (red), Met-133 (red), Val-136 (orange), Leu-139 (green), Leu-140 (yellow), Met159 (blue), Leu-239 (green), Leu-241 (magenta), Leu-242 (black), Val-243 (purple), and four potassium ions in the selectivity filter (green). M1-4 and $\mathrm{m} 2-4$ indicate the transmembrane domains 1-4 and 2-4 in opposing TASK-3 subunits, respectively. (B) Bottom/cytoplasmic view achieved by rotating the structure in A first $90^{\circ}$ then $35^{\circ}$ along its $x$-axes and $y$-axes, respectively. The surface rendering is transected through the putative anesthetic binding pocket (perforated red line) and the pore fenestration (blue perforated arrow). A $5 \AA$ ( $)$ scale bar is provided in A and B.
(LyTASK) homology model, residues 159 and 243 through 248 contribute to an "anesthetic binding pocket" (Bertaccini et al., 2014). Additionally, mutations at residues Leu-241, Leu-242, and Ser-155 (i.e., L241A, L242A, and S155W), near/in the "binding pocket" also modify halothane activation. TASK-3 and LyTASK share $47 \%$ amino acid identity, and the anesthetic-binding pocket is apparent in our TASK-3 homology structure (Fig. 1B and Supplemental Fig. 1). Val-136 in TASK-3, a hydrophobic residue, is conspicuous in its proximity to residues 159 and 243 and as a component of the anesthetic pocket's inner wall (Fig. 1B).

Excised, single-channel patch clamp studies demonstrate that anesthetics activate tandem pore channels through gating effects and not through surface expression or singlechannel conductance (Winegar and Yost, 1998; Patel et al., 1999). Although the mechanism of gating in TASK-3 is unknown, functional and crystallographic studies in related channels are notable. TREK-1 studies demonstrate a "C-type" gating mechanism involving residues near the selectivity filter. Both TRAAK and TREK-2 have been crystallized in presumed conducting and nonconducting conformations, with structural changes centered around the intracellular portions of transmembrane domains 2, 3, and 4 (Brohawn et al., 2014; Lolicato et al., 2014; Dong et al., 2015). Finally, hydrophobic residues in the pore of the TWIK-1 tandem pore channel (e.g., Leu-146) have a critical role in gating. Polar mutations at this residue cause channel activation, and modeling studies suggest the mechanism may be through effects on pore hydration essential for potassium conductance (Aryal et al., 2014). Residue Leu-122 in TASK-3 aligns with Leu-146 of TWIK-1; in our TASK-3 homology model, Leu-122 lines both the pore and the fenestration (Fig. 1).

In this study, we had three objectives. First, we determined that TCE activates TASK-3 and examined TASK-3 activation by a series of halogenated alcohols and related compounds to understand drug structural requirements for activation. Second, we hypothesized that mutants resistant to isoflurane activation would be similarly resistant to activation by TCE or by $N$-ethylmaleimide (NEM) modification of Cys-159, implying commonalities in their mechanisms of action. Third, we hypothesized that Val-136 contributes to anesthetic binding and transmits changes in the anesthetic-binding pocket to the pore residue, Leu-122. To test this hypothesis we mutagenized Val-136 and adjacent residues, as well as Leu-122, and studied effects on TASK-3 activation by TCE and in some cases isoflurane.

\section{Materials and Methods}

Molecular Biology. For the electrophysiology studies, rat TASK3, rat TASK-1, and LyTASK were cloned into variants of the pcDNA3.1 vector (Invitrogen, Carlsbad, CA). Rat TASK-1 has residues 312 to 331 deleted to promote surface expression (Renigunta et al., 2006). Directed missense mutations were introduced into cDNAs using the "QuikChange" method of mutagenesis (Phusion HF Mastermix; New England Biolabs, Ipswich, MA). All mutant cDNA were sequenced bidirectionally (MGH DNA Core Facility). The pYesMet25 yeast expression vector encoding yeast Trk1p or a nonfunctional Kir 2.1 "stuffer" cDNA was a generous gift from Prof. Dan Minor (University of California-San Francisco, CA). For our studies, we ligated wild-type TASK-3 or randomly mutagenized TASK3 cDNA into pYesMet25 employing a yeast Kozak sequence (AAAAA). 
Cell Culture, Transfection, and Ussing Chamber Studies. Fischer rat thyroid (FRT) epithelial cells were maintained in Ham's F-12 medium supplemented with 5\% fetal calf serum (Sigma-Aldrich, St. Louis, MO) at $37^{\circ} \mathrm{C}$ and $5 \% \mathrm{CO}_{2}$ in a humidified incubator. After trypsinization, the cells were cultured on semipermeable supports $\left(\sim 1.5 \times 10^{5}\right.$ cells per support; Corning Snapwell polycarbonate; $0.4 \mu \mathrm{m}$ pore size; $12 \mathrm{~mm}$ diameter; Fisher Scientific, Pittsburgh, PA) and were transfected in suspension with Lipofectamine 2000 (Invitrogen, Carlsbad, CA). The monolayers were studied 48 to 72 hours after transfection.

The electrophysiology methods were based on those of Sheppard et al. (1994). Before placing a study monolayer in the chamber, junction potentials were zeroed using a "dummy" membrane. A potassium gradient was applied across the study monolayer (basolateral $>$ apical), and the transepithelial voltage was clamped at $0 \mathrm{mV}$ (DVC-1000 amplifier; World Precision Instruments, Sarasota, FL). The apical bathing solution was (in $\mathrm{mM}$ ): $135 \mathrm{NaCl}, 1.2 \mathrm{MgCl}_{2}, 1.2 \mathrm{CaCl}_{2}$, 10 HEPES, 10 dextrose, pH 7.4 with $\mathrm{NaOH}$; the basolateral bathing solution was (in $\mathrm{mM}$ ): $135 \mathrm{KCl}, 1.2 \mathrm{MgCl}_{2}, 1.2 \mathrm{CaCl}_{2}, 10$ HEPES, 10 dextrose, $\mathrm{pH} 7.4$ with KOH. MES was used in lieu of HEPES in the pH 5.5 apical solution.

The Ussing chamber current signal was amplified and digitized $(10 \mathrm{~Hz})$ with a USB-6009 data acquisition board (National Instruments, Austin, TX) interfaced with an Apple Computer running Labview 8.5 software (National Instruments). The potassium current data were averaged every 1 second for analysis. By convention, positive current indicates positive charge flowing in the basolateral to apical direction; total current through the $1.2 \mathrm{~cm}^{2}$ monolayer is reported in the figures. Transepithelial voltage pulses $(+5 \mathrm{mV}$ and of 1 second duration), referenced to the apical surface, were applied intermittently to assess transepithelial resistance and monolayer integrity. Air was continuously bubbled through apical and basolateral solutions for mixing purposes.

All studies were conducted at room temperature $\left(22-24^{\circ} \mathrm{C}\right)$. Isoflurane, desflurane, sevoflurane, and halothane were applied using agent-specific variable bypass vaporizers in series with gas flow providing mixing bubbles to the Ussing chamber. A calibrated Datex Capnomac Ultima medical gas analyzer (GE Healthcare, Piscataway, $\mathrm{NJ}$ ) quantified the concentration in the gas phase (in \% atmosphere) and was converted to a solute concentration (in $\mathrm{mM}$ ) using methods described by Franks and Lieb (1988) with Ostwald partition coefficients $\left(22^{\circ} \mathrm{C}\right)$ of 1.57 (halothane), 1.21 (isoflurane), 0.42 (sevoflurane), and 0.29 (desflurane) (Hönemann et al., 1998).

Yeast Growth Studies. The potassium-sensitive trk1 $\Delta \operatorname{trk} 2 \Delta$ Saccharomyces cerevisiae yeast strain (R5421: ura3-52 his $3 \Delta 200$ leu2 $\Delta 1 \operatorname{trp} 1 \Delta 1$ ade2 $\operatorname{trk} 1 \Delta::$ HIS3 $\operatorname{trk} 2 \Delta::$ HIS3) was a generous gift from Prof. Richard Gaber (Northwestern University, Evanston, IL). Yeast plasmid DNA transformations were conducted using the Yeastmaker Yeast Transformation System 2 (Clontech, Mountain View, CA) or using the Frozen-EZ Yeast Transformation Kit (Zymo Research, Irvine, CA). Wild-type TASK-3, which fails to rescue the potassium-sensitive growth phenotype of $\operatorname{trk} 1 \Delta \operatorname{trk} 2 \Delta$ yeast, was optimized for functional expression in yeast by mutagenic polymerase chain reaction (PCR) (Cadwell and Joyce, 2006). The "yeast optimized" TASK-3 cDNA clone was transformed into yeast and was identified by growth on low-potassium agarose plates after replica plating.

"Yeast optimized" TASK-3 cDNA encodes the following six missense mutations: T17I, D27E, D32G, K79E, F202L, and F246Y. The optimized channel remains functional in FRT monolayers and provides potassium current levels similar to the wild-type TASK-3 channel (Supplemental Fig. 4). Additionally, it remains inhibited by extracellular acidic $\mathrm{pH}$, is activated by 2,2,2-tribromoethanol (TBE), and is inhibited by $\mathrm{m} 3$ muscarinic $\mathrm{G}$ protein coupled receptor ( $\mathrm{m} 3$ GPCR) activation ( $n=3$; data not shown). Supplemental Figure 5 demonstrates the potassium sensitivity of the trk $1 \Delta \operatorname{trk} 2 \Delta$ yeast strain and its phenotypic rescue by Trk1p and "yeast optimized" wild-type TASK-3 and less so by wild-type TASK-3 and a nonfunctioning Kir 2.1 "stuffer" potassium channel.
For these studies, after transformation and plating on highpotassium (100 mM KCl-added) -Ura/-Met/-His selective agar plates, five clones of trk $1 \Delta \operatorname{trk} 2 \Delta$ yeast transformed with each plasmid were combined, grown up in high-potassium selective medium, pelleted, washed with sterile water to remove excess potassium, and resuspended in low-potassium (no added potassium) or high-potassium -Ura/-Met/-His liquid media (US Biologic, Salem, MA) at a $650 \mathrm{nM}$ light absorbance (A650) of $\sim 0.01$. After dilution, cells were cultured at $30^{\circ} \mathrm{C}$ in 96-well format with continuous shaking on a Titer Plate Shaker (Model 4625; Laboratory-Line Instruments/Thermo Fisher Scientific, Waltham, MA). Growth was measured intermittently by A650 nm analysis using a SpectraMAX 190 plate reader (Molecular Devices, Sunnyvale, CA).

For TBE selection studies, yeast expressing "yeast optimized" wildtype TASK-3 and "yeast optimized" TASK-3 V136E were cultured in high-potassium selective synthetic liquid medium (-Ura/-Met/-His) at $30^{\circ} \mathrm{C}$ to a $600 \mathrm{nM}$ light absorbance (A600) 1.0 (P330 Nanophotometer; Implen, Westlake Village, CA). Yeast were pelleted and washed, resuspended, mixed 1:1 (wild-type:V136E), diluted to an A600 of $\sim 0.01$, and regrown in a $5-\mathrm{ml}$ volume liquid culture in the presence or absence of $1 \mathrm{mM}$ TBE at $30^{\circ} \mathrm{C}$ in low-potassium selective synthetic medium (-Ura/-Met/-His). After growth to an A600 1.0, yeast were pelleted by centrifugation, plasmid DNA was recovered (QIAprep Spin Miniprep kit, Qiagen, Germantown, MD), and the sequence flanking residue-136 was amplified by PCR. After gel purification, PCR fragments were subjected to MiSeq Next Generation Sequencing $(2 \times 150 \mathrm{bp}$; Illumina, San Diego, CA $)$ in the MGH DNA Core, and custom computer software script written in Python was used to analyze the sequencing data and to count the frequency of Val-136 relative to Glu-136 in each sample.

Graphing, Statistical Analysis, Chemical Structure Analysis and Homology Model. Microsoft Excel (Microsoft, Redmond, WA), Prism 5.0b (GraphPad Software, La Jolla, CA), and Adobe Illustrator (Adobe Systems, San Jose, CA) were used for data/statistical analysis, graphing, regression, and figure preparation. A one-way analysis of variance and a post hoc Bonferroni multiple comparison test or a Student's $t$ test were used to compare group means (as indicated in the figure legends or text). Molecular structures, graphics, and analyses were performed with the UCSF Chimera software package and ChemDoodle/ChemDoodle 3D (iChemLabs, Somerset, NJ). Chimera software was developed by the Resource for Biocomputing, Visualization, and Informatics at the University of California-San Francisco (supported by NIGMS P41-GM103311) (Pettersen et al., 2004). Molecular volume were calculated with Chimera; $X \log P$ and polarizability were estimated using ChemDoodle (iChemLabs). The TASK-3 homology (Fig. 1) was developed as previously described and is based on the PDB 3UKM (TWIK-1 channel) structure (Miller and Long, 2012; Chokshi et al., 2015).

Reagents. All chemicals and supplies and reagents were obtained from Sigma-Aldrich or Fisher Scientific. NEM, carbon tetrabromide, and carbon tetraiodide were solubilized in dimethylsulfoxide (DMSO). TASK-3 function is minimally affected by DMSO at final bath concentrations up to $2 \%$ (normalized current of $100 \% \pm 1.1 \%$; $n=$ 3 ; and concentration-response studies remained at or below this level.

\section{Results}

Halogenated Ethers, Alcohols, and Alkanes Activate TASK-3 Potassium Channel Function. Rat TASK-3 was transiently expressed in FRT cell monolayers, which provides a large, positive, $\mathrm{pH}$-inhibited transepithelial potassium current not otherwise present in FRT monolayers. The TASK-3 potassium current was activated by a range of halogenated compounds (Figs. 2, 3, and 4) including 2,2,2-trichloroethanol (TCE). Ethanol (without halogenation) (Fig. 3) provided no activation at similar 
A

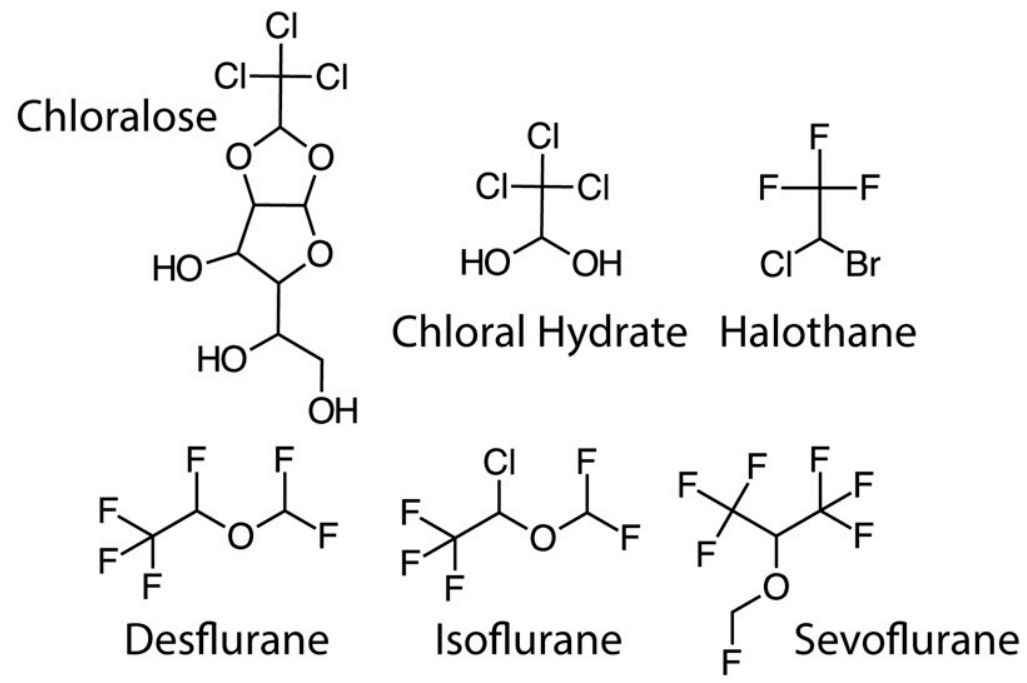

B<smiles>OCC(Br)(Br)Br</smiles><smiles>OCC(Cl)(Cl)C(Cl)(Cl)C(F)(F)F</smiles><smiles>OCC(F)(F)C(F)(F)C(F)(F)F</smiles>

$\mathrm{EtOH}$<smiles>ClC(I)(I)I</smiles><smiles>BrC(Br)(Br)Br</smiles><smiles>ClC(Cl)(Cl)[Ge](Cl)(Cl)Cl</smiles><smiles>OC(C(F)(F)F)C(F)(F)F</smiles>

C

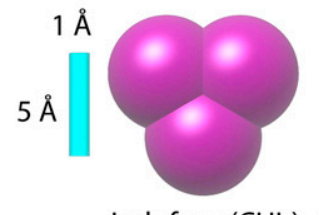

lodoform $\left(\mathrm{CHI}_{3}\right)$ $232.0 \mathrm{~cm}^{3} / \mathrm{mol}^{3}$ $18.072 \AA^{3}$ XlogP 3.688

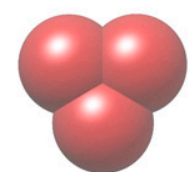

Bromoform $\left(\mathrm{CHBr}_{3}\right)$ $154.7 \mathrm{~cm}^{3} / \mathrm{mol}$ $11.967 \AA^{3}$ XlogP 3.088

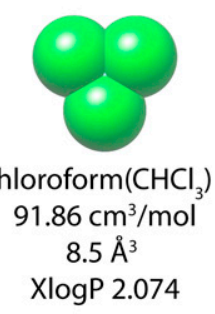

$\begin{aligned} & 8.5 A^{3} \\ & X \log P \\ & 2.074\end{aligned}$

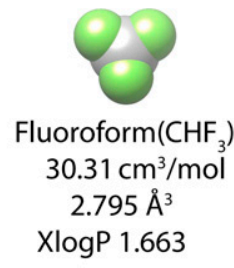

$\mathrm{X} \log \mathrm{P} 1.663$<smiles>CCO</smiles>

Fig. 2. (A) Chemical structure of clinically relevant agents used in this study. (B) Chemical structures of some halogenated ethanol and methane drugs/compounds used in this study: TBE, 2,2,2-tribromoethanol; TCE, 2,2,2-trichloroethanol; TFE, 2,2,2-trifluoroethanol; EtOH, ethanol; CI ${ }_{4}$, carbon tetraiodide; $\mathrm{CBr}_{4}$, carbon tetrabromide; $\mathrm{CCl}_{4}$, carbon tetrachloride; and HFP, 1,1,1,3,3,3-hexafluoro-2-propanol. (C) Illustration of relative sizes of iodoform $\left(\mathrm{CHI}_{3}\right)$, bromoform $\left(\mathrm{CHBr}_{3}\right)$, chloroform $\left(\mathrm{CHCl}_{3}\right)$, fluoroform $\left(\mathrm{CHF}_{3}\right)$, and 1,1,1,3,3,3-hexafluoro-2-propane. A $5 \AA$ ( $(\AA)$ scale bar (in light blue), estimated volume (in $\mathrm{cm}^{3} / \mathrm{mol}$ ), average molecular polarizability (in $\AA^{3}$ ), and calculated lipophilicity $(X \log P$ ) are provided.

concentrations, suggesting it is the halogenated 2-carbon on the various ethanol derivatives that engages the channel. Consistent with this, carbon tetrabromide $\left(\mathrm{CBr}_{4}\right)$ and carbon tetrachloride $\left(\mathrm{CCl}_{4}\right)$ both activate TASK-3 (Fig. 3). Of all the agents studied, $\mathrm{CBr}_{4}$ had the highest potency $\left(\mathrm{EC}_{50} \sim 16 \mu \mathrm{M}\right)$ and efficacy (normalized current of $\sim 296 \%$ ) for wild-type TASK-3 activation (Fig. 3). Clinically relevant agents chloral hydrate, halothane, isoflurane, sevoflurane, and desflurane all activate TASK-3; and, of these, halothane and desflurane were the most and least effective, respectively (Fig. 4). Interestingly, 1,1,1,3,3,3hexafluoropropanol (HFP; Fig. 2), a component of sevoflurane, activates like sevoflurane (Fig. 4 ) but with $\sim 5$-fold lower potency. Notably, the largest agents, carbon tetraiodide and $\alpha$-chloralose, as well as the smallest (e.g., ethanol, 2-chloroethanol, 2,2,2- trifluoroethanol, isoamyl alcohol), failed to activate or minimally activated TASK-3 (Figs. 3 and 4).

The TASK-3 homologs LyTASK and TASK-1 were both activated by halogenated ethanols with a greater efficacy than that of wild-type TASK-3 (Supplemental Fig. 2; LyTASK: $708 \% \pm 47 \%[n=5]$ normalized current at $5 \mathrm{mM}$ TBE and $1054 \% \pm 248 \%[n=4]$ at $20 \mathrm{mM}$ TCE; TASK-1: $3531 \% \pm$ $2845 \%[n=3$ ] at $4.4 \mathrm{mM} \mathrm{TBE}$ ). The concentration-response curves for both channels, however, were right-shifted relative to wild-type TASK-3, suggesting a lower binding affinity to the activating compounds relative to TASK-3 (Supplemental Fig. 2D). The very small basal currents provided by TASK-1 (1.6 \pm $1.1 \mu \mathrm{A} ; n=3$ ) contributed significant variability to our TASK1 results. 


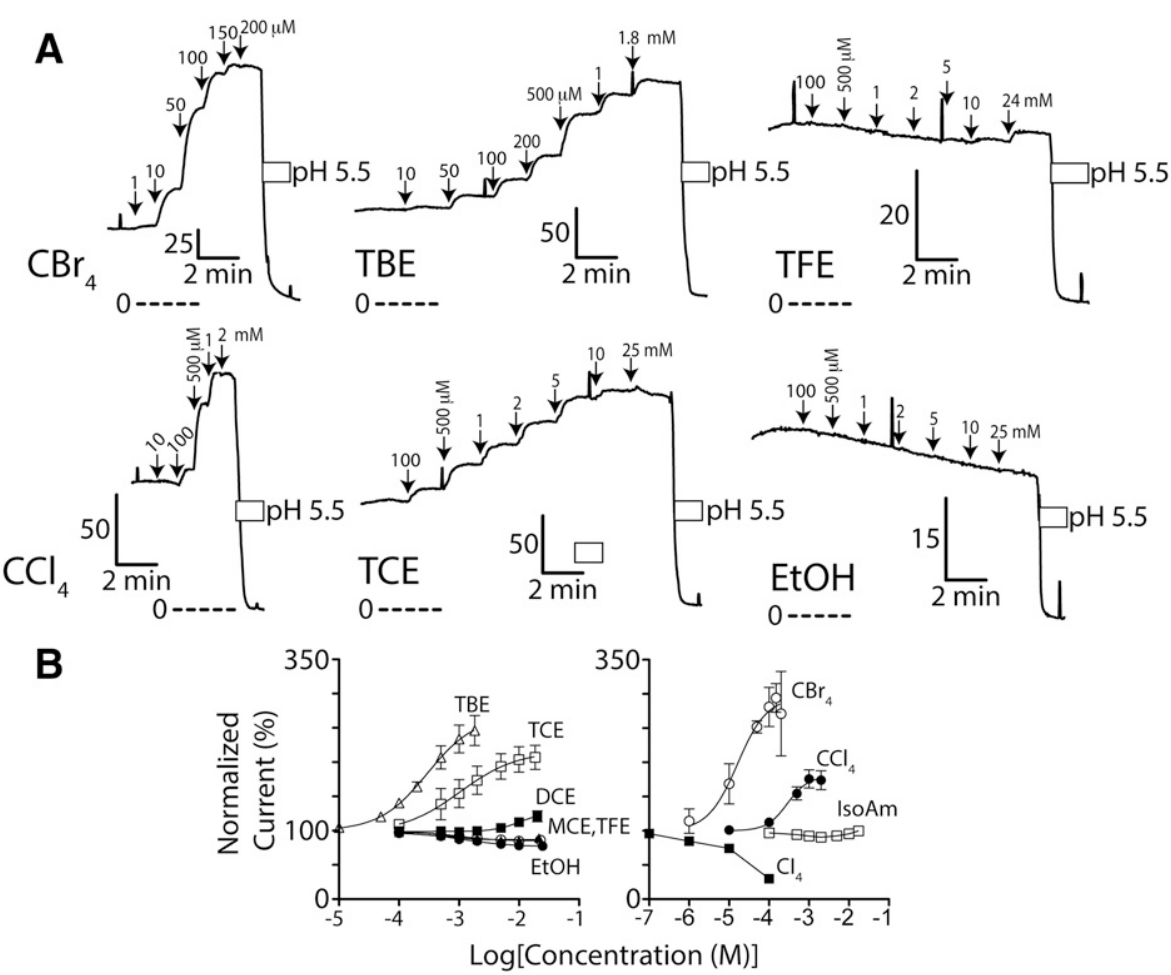

Fig. 3. TASK-3 channel function is activated by brominated and chlorinated methanes and alcohols. (A) Ussing chamber potassium current records using Fischer rat thyroid cell monolayers expressing wild-type TASK-3 channels. Downward arrows indicate study compound application; perforated lines are zero current level; L-shaped bars are current (in $\mu \mathrm{A}$ ) and time scale; and white bars are acidic $\mathrm{pH}$. In each, transmonolayer voltage was clamped at $0 \mathrm{mV}$; intermittent $+5 \mathrm{mV}$ pulses assess membrane integrity. (B) Normalized, steady-state concentrationresponse of wild-type TASK-3 to EtOH, isoamyl alcohol, TFE (2,2,2-trifluoroethanol), MCE (2-chloroethanol), DCE (2,2-dichloroethanol), TCE, TBE, $\mathrm{CCl}_{4}, \mathrm{CBr}_{4}$, and $\mathrm{CI}_{4}$ (carbon tetraiodide). $n=$ from 3 to 8 for each \pm S.D. Data were normalized to baseline current. Data for TCE, TBE, $\mathrm{CCl}_{4}$, and $\mathrm{CBr}_{4}$, which caused clear activation, were fit with the following: $Y=100+$ $\left(I_{\max }-100\right) /\left(1+10^{\wedge}\left(\left(\log _{10} \mathrm{EC}_{50}-X\right)^{*}\right.\right.$ Hill Slope)) in which $I_{\max }=$ maximum normalized current, and $X=\log _{10}$ [activating agent]. TCE, TBE, $\mathrm{CCl}_{4}$, and $\mathrm{CBr}_{4}$ data, respectively, for $I_{\max }$ (in \%; 95\% confidence): 215 (196-234), 267 (240294), 180 (163-196), and $296(245-346) . \mathrm{EC}_{50}$ (in $\mu \mathrm{M})$ : 1077 (647-793), 295 (195-447), 289 (170$492)$, and 16 (7-37). Hill Slope: $1.0(0.5-1.4), 1.1$ $(0.8-1.4), 1.7(0.7-2.7)$, and $1.2(0.2-2.2)$. Data points for other nonactivating agents are connected with a straight line.
Amino Acids Both in the Anesthetic Binding Pocket and in the Pore Are Required for TASK-3 Activation. Val-136 resides in close proximity to Met-159 and lines a putative anesthetic-binding pocket in TASK-3 (Fig. 1). Mutagenesis of residue-136 to various residues impairs TCE activation (Fig. 5; Supplemental Fig. 3). Because introduction of a charged aspartate residue in the putative anesthetic- binding pocket should impair TASK-3 interactions with the hydrophobic trichloromethane substituent of TCE, we undertook scanning aspartate mutagenesis of residues adjacent to 136 in the primary amino acid sequence (132-140). Residues 133, 139, and 140, each approximately one helical turn from 136 and predicted to line the anesthetic pocket (Figs. 1 and 5C), when mutated modify TCE activation.
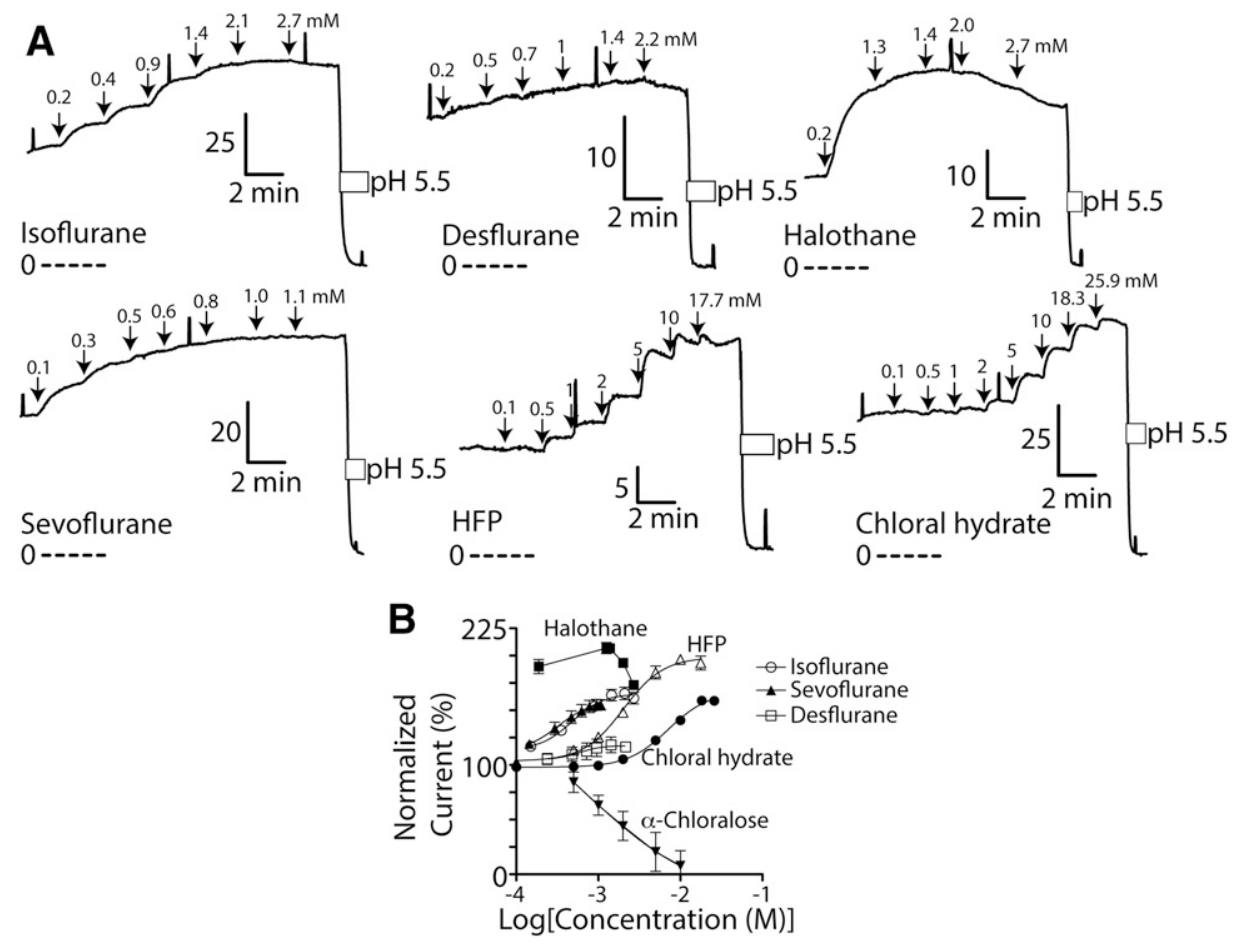

Fig. 4. TASK-3 channel function is activated by multiple clinically relevant halogenated agents. (A) Ussing chamber potassium current records using Fischer rat thyroid cell monolayers expressing wild-type TASK-3 channels. Data were collected as described in Fig. 3 with $n=3$ to $5 \pm$ S.D. for each. (B) Concentration-response for halothane, isoflurane, desflurane, sevoflurane, hexafluoropropanol (HFP), chloral hydrate, and $\alpha$-chloralose. Data points were fitted as in Fig. 3. Chloral hydrate, isoflurane, desflurane, sevoflurane, and HFP data, respectively, for $I_{\max }$ (in \%; 95\% confidence): 165 (161-170), 169 (161-176), 119 (109-129), 164 (150-177), and 200 (194-206). $\mathrm{EC}_{50}$ (in $\left.\mu \mathrm{M}\right): 7141$ (63867986), 363 (283-466), 433 (179-1047), 265 (181-38) and 1942 (1683-2241). Hill Slope: 2.0 (1.7-2.3), $1.5(1.0-2.0), 1.7(-0.8$ $4.3), 1.4(0.8-2.1)$, and $1.6(1.3-1.9)$. 
A
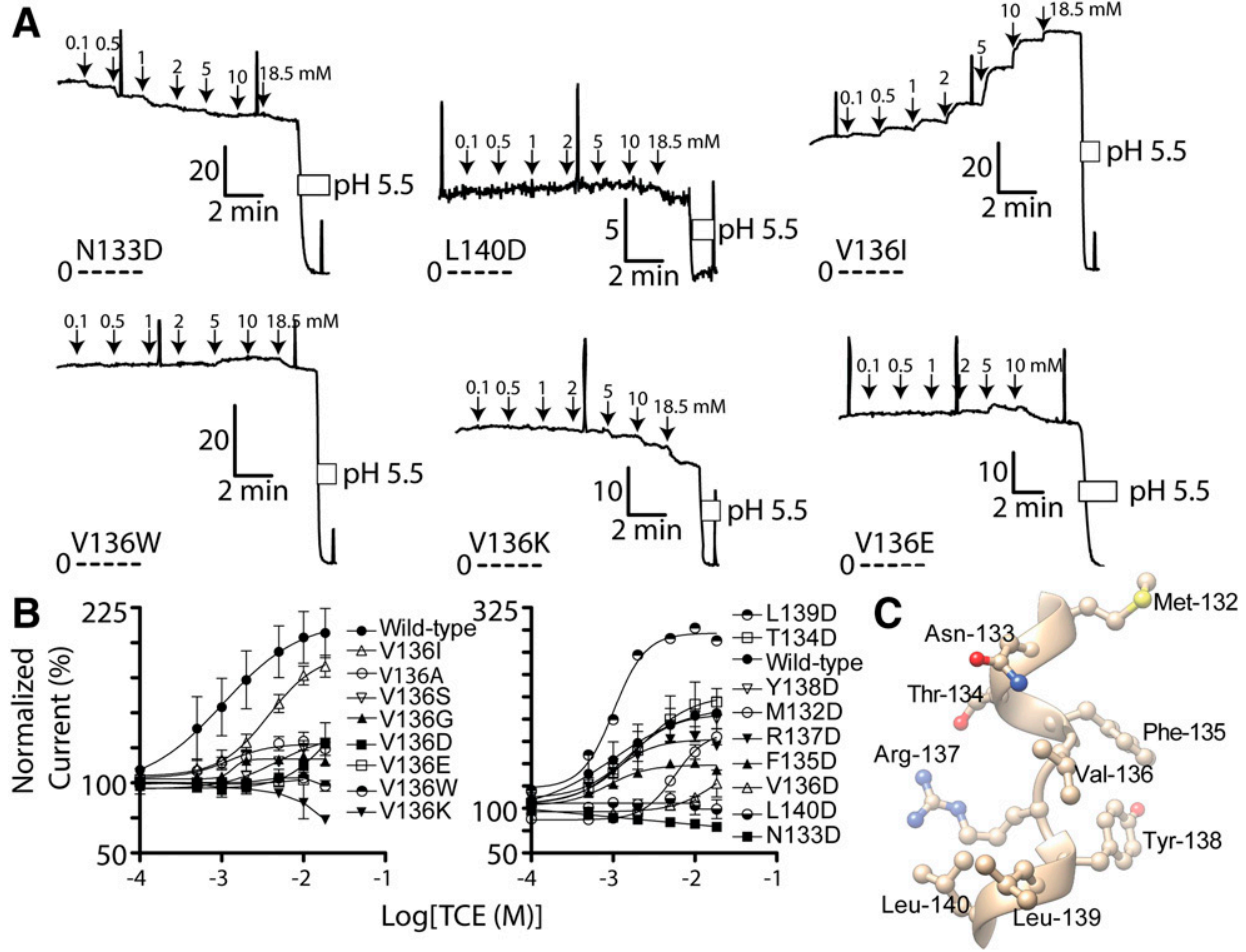

Fig. 5. Effects of TASK-3 residue-136 and adjacent residue mutagenesis on 2,2,2-trichloroethanol (TCE) activation. (A) Representative current records from FRT monolayers expressing TASK-3 N133D, L140D, V136I, V136W, V136K, and V136E. Details of currents records are as described in Fig. 3. (B) Compiled TCE concentration-response for multiple TASK-3 mutants. Data points were fitted as described in Fig. 3. For mutants V136A, V136G, V136I, V136S, V136D, respectively, $I_{\max }: 129 \%$ (95\% CI, 123-134), 117\% (95\% CI, 115-120), 198\% (95\% CI, 177-220), 130\% (95\% CI, 124-137), 139\% (95\% CI, 63-216). $\mathrm{EC}_{50}$ (in $\left.\mu \mathrm{M}\right)$ : 977 (566-1687), 1188 (841-1679), 3867 (2308-6477), 4900 (3478-6904), 13,300 (2290-77260). Hill Slope: 1.3 (0.4-2.1), 3.0 (0.25.8), 1.2 (0.8-1.6), 2.1 (1.0-3.1), 2.7 (-2.7-8.1). For mutants M132D, T134D, F135D, R137D, Y138D, L139D, respectively, $I_{\max }$ : 180\% (95\% CI, 162-199), $251 \%$ (95\% CI, 239-264), $149 \%$ (95\% CI, 146-153), $178 \%$ (95\% CI, 173-184), $211 \%$ (95\% CI, 201-221), 300\% (95\% CI, 280-320). EC (in $\mu \mathrm{M}$ ): 6084 (45878070), 1297 (1036-1623), 782 (636-961), 888 (732-1077), 1351 (1035-1762), 869 (661-1143). Hill Slope: 3.2 (0.7-5.6), 1.3 (0.9-1.6), 1.6 (1.0-2.1), 1.5 (1.01.9), $1.1(0.8-1.4), 1.7(0.9-2.5)$. (C) Relevant segment of the TASK-3 homology model (residues Met-132 through Leu-140) to illustrate the predicted three-dimensional relationship of the residue side chains.

Because Leu-122 resides on the same transmembrane domain as Val-136 (Fig. 1) and because an equivalent residue in the TWIK-1 channel pore (Leu-146) has a putative role in gating, we studied the effect of Leu-122 mutation on TASK-3 activation. Interestingly, the L122D mutation completely eliminates activation by both isoflurane and TCE (Fig. 6 and Supplemental Fig. 3). From prior work, we know mutations at TASK-3 residue-159 (e.g., M159W) impair activation by halogenated volatile anesthetics such as isoflurane (AndresEnguix et al., 2007; Conway and Cotten, 2012). In the current study, we observed that M159W is similarly resistant to TCE activation (Fig. 6 and Supplemental Fig. 3).

NEM modification of Cys-159 in the TASK-3 M159C mutant causes marked channel activation. NEM is an uncharged, membrane-permeant, thiol-selective alkylating agent with minimal effects on wild-type TASK-3 function (Conway and Cotten, 2012). We studied the effect of L122D and V136E on this process by preparing the following double mutants, L122D/M159C and V136E/M159C, and quantifying the extent of activation by $5 \mathrm{mM}$ NEM (Fig. 7). L122D and V136E mutations both impaired $5 \mathrm{mM}$ NEM activation; the smaller residual response to NEM suggest Cys-159 is still alkylated in both double mutants (Fig. 7). Like wild-type TASK-3, the function of single mutants TASK-3 L122D and V136E are minimally impacted by $5 \mathrm{mM}$ NEM exposure of the same duration (L122D: $100 \% \pm 1 \%$ normalized current, $n=3$; V136E: $89 \% \pm 4 \%, n=3)$.
TASK-3 V136E, Unlike L122D and N133D, Retains Sensitivity to m3 Muscarinic GPCR Inhibition. TASK-3 channels are inhibited by $\mathrm{G} \alpha_{\mathrm{Q} / 11}$-coupled GPCRs, and several anesthetic-resistant mutant TASK-3 channels are resistant to

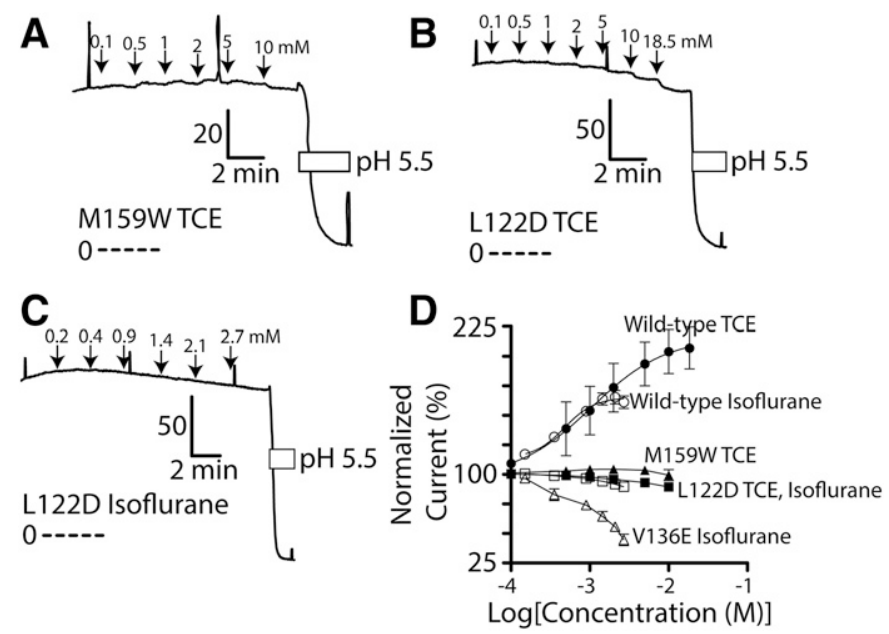

Fig. 6. TASK-3 mutants L122D, V136E, and M159W disrupt TCE and isoflurane activation. (A-C) Currents records from FRT monolayers expressing TASK-3 M159W and L122D and their response to TCE or isoflurane. Details of currents records are as described in Fig. 3. (D) Compiled TCE concentration-response for TASK-3 M159W, L122D, and V136E. Wild-type TASK-3 data are from Figs. 3 and 4. Data points for M159W, L122D, and V136E are connected by a straight line. 

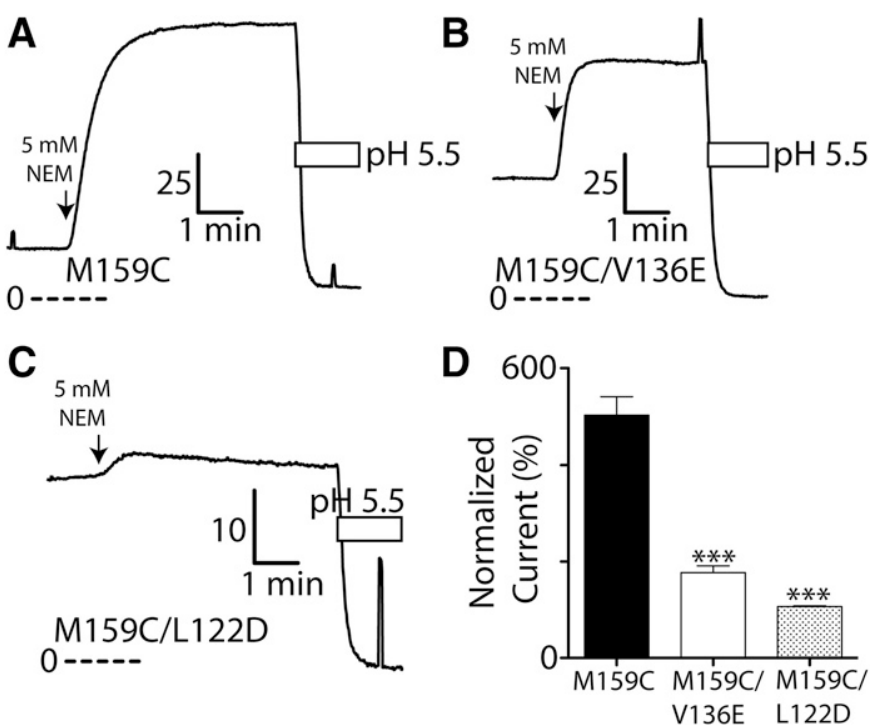

Fig. 7. TASK-3 activation after Cys-159 covalent modification is impaired by L122D and V136E. Currents records from FRT monolayers expressing TASK-3 M159C (A), M159C/V136E (B), and M159C/L122D (C) exposed to $5 \mathrm{mM}$ NEM, a cysteine-selective alkylating agent. Details of currents records are as described in Fig. 3. (D) Peak steady-state normalized current following $5 \mathrm{mM}$ NEM modification for M159C, M159C/V136E, and M159C/L122D. Data are fitted as in Fig. $3 ; n=$ at least $3 \pm$ S.D. for each. $* * * P<0.001$, statistical significance relative to TASK-3 M159C as determined by a one-way ANOVA and a post hoc Bonferroni multiple comparison test.

this GPCR regulation, including M159W, NEM-modified M159C, and TASK-3/TREK-1 channels in which residues 243 to 248 (VLRFLT) are mutated to GDWLRV (Talley and Bayliss, 2002; Conway and Cotten, 2012). To explore the effects of L122D, N133D, V136E, and L140D mutations on TASK-3 GPCR regulation, we studied each mutant's functional response to activation of a coexpressed m3 GPCR. Carbachol, an m3 GPCR agonist, inhibited potassium currents in V136E/m3 GPCR and in wild-type TASK-3/m3 GPCR-expressing monolayers to a similar extent (Fig. 8). Currents through TASK-3 L122D and N133D channels, however, were completely resistant to GPCR regulation although they remained sensitive to inhibition by extracellular $\mathrm{pH}$ (Fig. 8). The L140D mutant was somewhat resistant to GPCR regulation.

TBE Selects for Wild-Type TASK-3 over V136E TASK-3 Expressed in trk1 $\Delta$ trk2 $\Delta$ Potassium-Sensitive Yeast. Saccharomyces cerevisiae yeast lacking Trk1p and Trk2p potassium transporters (trk1 1 trk2 $2 \Delta$ ) requires supplemental potassium (e.g., $100 \mathrm{mM} \mathrm{KCl}$ ) for growth. Heterologous potassium channel expression, however, rescues their phenotype to permit growth in low-potassium media (Supplemental Fig. 5), which enables their use in high-throughput pharmacologic and mutagenic potassium channel screens (Nakamura and Gaber, 1998; Bagriantsev and Minor, 2013). To validate our electrophysiology findings in a different system, $\operatorname{trk} 1 \Delta \operatorname{trk} 2 \Delta$ yeast were transformed with "yeast optimized" (see Materials and Methods) wild-type TASK-3 (containing Val-136) or V136E TASK-3 cDNA and mixed in 1:1 ratio (wildtype:V136E) for culture in low potassium media in the presence or absence of $1 \mathrm{mM}$ TBE. After growth to an A600 > 1.0, plasmid DNA was isolated from the cultures and the relative frequency of each TASK-3 variant (Val-136 or Glu-136 containing) were counted by next-generation sequencing. Supplemental Fig. 6 shows that yeast grew more slowly in the presence of $1 \mathrm{mM}$ TBE taking 5.7 versus 2.8 days to reach an A600 > 1.0. Figure 9, however, shows that TBE enriched the relative frequency of wild-type TASK-3 over that of TASK-3 V136E.

\section{Discussion}

In this study we measured the concentration-response of several halogenated alcohols, alkanes, and ethers on TASK-3 channel function and identified several new potent and effective TASK-3, LyTASK, and TASK-1 activating compounds (e.g., TCE, TBE, $\mathrm{CCl}_{4}$, and $\mathrm{CBr}_{4}$ ). Guided by a TASK-3 homology model, we identified several residues (e.g., 133, 136, 139, and 140) predicted to line a putative anesthetic binding pocket-first described in the LyTASK potassium channel homolog-that, when mutated, modify TCE and isoflurane activation of TASK3 or NEM activation of TASK-3 M159C. Finally, we identified Leu-122, a putative gating residue in the channel pore, that when mutated (L122D) renders TASK-3 resistant to activation by TCE, isoflurane, and NEM and to inhibition by activated m3 GPCRs.

Drug Attributes Required for TASK-3 Activation. We determined halogenated agents (alcohols, alkanes, and ethers) activate TASK-3. It is likely the halogenated 2-carbon of ethanolic agents that engages the channel to promote activation (Fig. 10) and not the hydroxylated 1-carbon, because nonhalogenated ethanol fails to activate TASK-3 and because both $\mathrm{CBr}_{4}$ and $\mathrm{CCl}_{4}$ cause TASK-3 activation (Fig. 3). The degree (e.g., mono-, di-, versus trichloroethanol) and type of halogenation (e.g., fluorine, chlorine, versus bromine) at the 2-carbon influences activation potency and efficacy (Fig. 3).

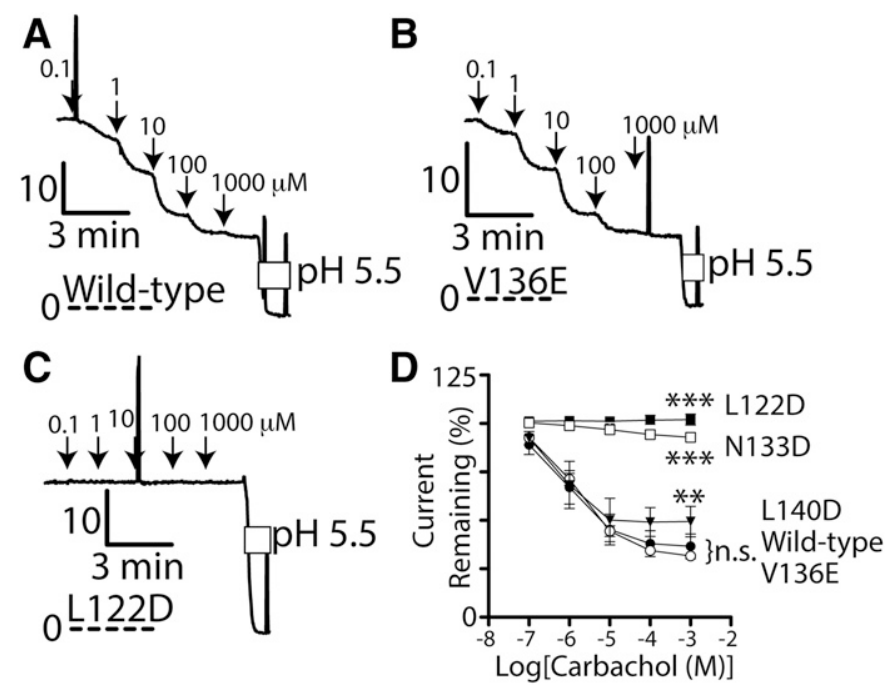

Fig. 8. TASK-3 L122D is resistant to $\mathrm{m} 3 \mathrm{GPCR}$ regulation. (A) Currents records from FRT monolayers coexpressing $\mathrm{m} 3 \mathrm{GPCR}$ and wild-type (A), V136E (B), and L122D (C) TASK-3 channels exposed to increasing concentrations of carbachol (downward arrows). Details of currents records are as described in Fig. 3. (D) Compiled carbachol concentration-response for L122D, N133D, L140D, wild-type, and V136E TASK-3 channels; $n=$ at least $3 \pm$ S.D. for each data point. $* * * P<0.001$ and $* * P<0.01$, statistical significance relative to wild-type and V136E TASK-3 at $1 \mathrm{mM}$ carbachol as determined by a one-way ANOVA and a post hoc Bonferroni multiple comparison test. 


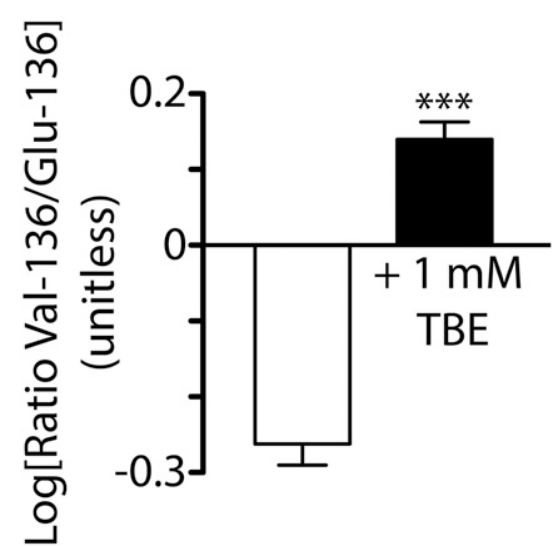

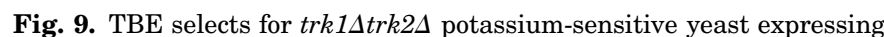
"yeast optimized" wild-type (i.e., Val-136) TASK-3 over those expressing "yeast optimized" TASK-3 V136E (i.e., Glu-136) mutant. The $\log _{10}$ ratio of residue-136 TASK-3 cDNA variants (Val-136/Glu-136) transformed yeast growth (quantified by A600) grown in the presence of DMSO (vehicle) or $1 \mathrm{mM}$ TBE. Bars indicate mean \pm S.D.; $n=4$ samples each. TASK-3 cDNA was optimized for yeast functional expression as discussed in Materials and Methods. Relative frequency of each residue-136 variant in samples was counted by Illumina MiSeq DNA sequencing. Sequence counts (Val136/Glu-136) for all samples grown without TBE: 7541/11073, 4981/7036, $8157 / 10641$, and $11265 / 15075$; sequence counts for samples grown with $1 \mathrm{mM}$ TBE: $10630 / 5547,14113 / 7440,14633 / 8785$, and 14104/7630. $* * * P<$ 0.001 , statistical significance of Val-136/Glu-136 ratios from samples grown in the presence of $1 \mathrm{mM}$ TBE relative to those grown in DMSO vehicle alone using an unpaired, two-tailed Student's $t$ test.

Halogenation has at least three effects on the molecule: 1) increased volume, 2) increased polarizeability, and 3) increased hydrophobicity.

We used fluoro-, chloro-, bromo-, and iodoform chemical structures as surrogates to estimate the physicochemical properties of the halogenated 2-carbon substituent (Fig. 2C). From these, we speculate a 2 -carbon size between 30.31 (from fluoroform) and $232.0 \mathrm{~cm}^{3} / \mathrm{mol}$ (from iodoform) may be required to support TASK-3 activation (Fig. 2). Like the alcohols (Fig. 10), we speculate only a portion of the larger halogenated ether/alkane molecules binds within the TASK-3 "binding cavity," such as the hexafluoropropane substituent of sevoflurane (Fig. 2). The increased potency of halogenated methanes relative to the similarly halogenated ethanols (e.g., $\mathrm{EC}_{50}$ of 1077 versus $289 \mu \mathrm{M}$ for TCE versus $\mathrm{CCl}_{4}$ and 295 versus $16 \mu \mathrm{M}$ for TBE versus $\mathrm{CBr}_{4}$ ) may be due to symmetry, as the methanes engage the channel in an identical manner regardless of orientation. An alcohol must orient itself to enter the binding site; additionally, the 1-carbon hydroxyl may favor solvation in an aqueous environment (Fig. 10). Interestingly, chloral hydrate (Fig. 2), which contains an additional hydroxyl group, is less potent than TCE $\left(\mathrm{EC}_{50}\right.$ of 7141 versus $1077 \mu \mathrm{M}$, respectively).

Increased substituent polarizeability may also enhance potency: for example, $\mathrm{CBr}_{4}$ is more potent than $\mathrm{CCl}_{4}\left(\mathrm{EC}_{50}\right.$ of 16 versus $289 \mu \mathrm{M}$ ), and TCE is more potent than the similarly sized and similarly effective TASK-3 activator HFP $\left(\mathrm{EC}_{50}\right.$ of 1077 versus $\left.1942 \mu \mathrm{M}\right)$, despite its increased hydrophobicity (XlogP 3.088 versus 3.418; Fig. 2C). As reference, bromoform, chloroform, and hexafluoropropane have estimated polarizabilities of $12.0,8.5$, and $6.6 \AA^{3}$ (Fig. 2C). Crystallographic studies of protein-halogenated anesthetic complexes demonstrated polarization of the hydrophobic anesthetic molecules to accommodate polar and nonpolar interactions within amphiphilic binding cavities (Bertaccini et al., 2007). The putative cavity in TASK-3 (Supplemental Fig. 1) is largely hydrophobic with some polar characteristics provided by Ser-155 and Met-159. The hypnotic potency of several halogenated compounds used in this study are shown in Supplemental Table 1 for reference.

From this data and the work of others, it is known that several anesthetic agents fail to activate TASK-3 including ethanol, xenon, nitrous oxide, propofol, etomidate (personal observation), cyclopropane, carbon tetraiodide, and $\alpha$-chloralose (Gruss et al., 2004; Putzke et al., 2007). Given our current findings, we speculate these compounds may lack overall volume or lack a hydrophobic, polarizeable substituent of suitable volume (e.g., tribromo-methyl of TBE) that can engage in interactions necessary for activation. The ethanol 2-carbon (methane $=38 \mathrm{~cm}^{3} / \mathrm{mol}$ ), xenon $\left(43 \mathrm{~cm}^{3} / \mathrm{mol}\right)$, nitrous oxide (35 $\left.\mathrm{cm}^{3} / \mathrm{mol}\right)$, and cyclopropane $\left(63 \mathrm{~cm}^{3} / \mathrm{mol}\right)$ are likely too small; propofol $\left(639.5 \mathrm{~cm}^{3} / \mathrm{mol}\right)$ and etomidate $\left(835.5 \mathrm{~cm}^{3} /\right.$ mol) are likely too large (Firestone et al. 1986). Carbon tetraiodide may fail to activate due to size constraints; however, aqueous solubility, degradation, and/or simultaneous TASK-3 inhibition may also be a factor. Although $\alpha$-chloralose possesses a trichloromethane substituent sufficient for activation in other contexts (e.g., carbon tetrachloride, chloroform, trichloroethanol, and chloral hydrate), the large, water-soluble carbohydrate substitution may sterically impair access to the activation site, favor hydration in water, and/or cause TASK-3 inhibition.

The Anesthetic-Binding Site. We identified several missense mutants that impair TASK-3 activation at residues 122 , 133, 136, 140, and one mutant that enhances it (residue-139) (Fig. 5 and Supplemental Fig. 3). The simplest interpretation is that these residues have a direct or near direct role in anesthetic binding, and that the mutations that diminish activation diminish interactions with the anesthetic and/or introduce a residue that sterically or electrostatically excludes it. Also and alternatively, introduction of a polar residue may favor water occupancy of the binding site over an anesthetic. Enhancing mutations might have the opposite effect.

Mutations, however, may also have an indirect, allosteric effect, and modify the binding site from afar, and/or modify

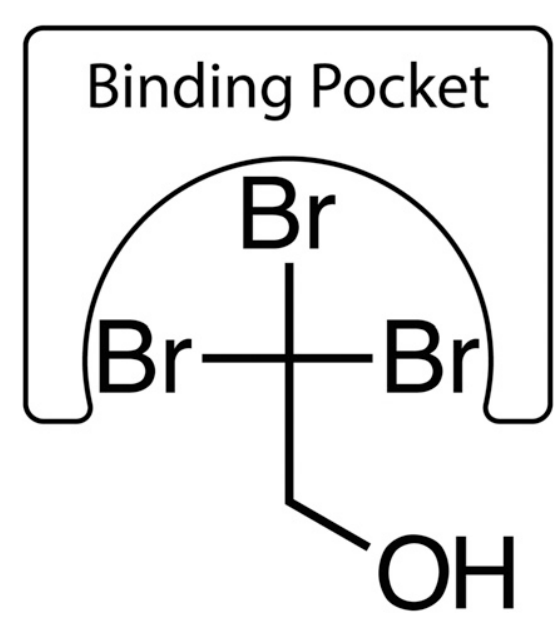

Fig. 10. Cartoon model of proposed 2,2,2-tribromoethanol interaction with the TASK-3 anesthetic binding site. The 2 -carbon and its bromine atoms interacts with the anesthetic-binding site with the 1-carbon hydroxyl excluded. 
channel gating (i.e., its open-state probability) such that the functional consequences of binding are minimized or enhanced. For example, a channel that is "locked open" cannot be further activated, whether it is binding an anesthetic or not. Crystal structures of TRAAK channels with a single activating mutation in the P-loop alpha helix have significant alterations in protein structure remote from the mutation site (Lolicato et al., 2014).

The results of the TASK-3 L122D mutation are novel, as mutations in this region of TASK-3 have never been shown to modify anesthetic activation. Leu-122 in our homology model resides in the TASK-3 pore adjacent to the fenestration and in proximity to Leu-239 of the adjacent subunit (Fig. 1). Polar mutations at an equivalent residue in TWIK-1 (Leu-146) activate TWIK-1, and molecular dynamic studies suggest mutations here alter pore hydration and/or alter lipid access into the pore through the fenestration (Aryal et al., 2014, 2015). Hydrophobic gating is a property of hydrophobic nanopores, and hydration allows ions to conduct through the pore; and, as others have proposed, anesthetic agents may modify hydrophobic gating (Roth et al., 2008).

The L122D mutation may eliminate hydrophobic gating effects and thus anesthetic effects on hydrophobic gating. TREK-2 binds norfluoxetine in its hydrophobic fenestration (Dong et al., 2015). Likewise, TASK-3 may also bind anesthetics in its fenestration (Poulsen and Nissen, 2012), which would prevent lipids from accessing and blocking the pore; and L122D may disrupt both anesthetic fenestration binding and lipid pore entry (Brohawn et al., 2014). However, TASK-3 L122D is resistant to activation by both anesthetics and NEM modification of Cys-159 and to inhibition by m3 GPCR activation, all similar to that seen previously in the TASK-3/TREK-1 mutant (Talley and Bayliss, 2002; Conway and Cotten, 2012).

These observations suggests that Leu-122 has a critical role in TASK-3 gating, and we speculate that the L122D mutation stabilizes an open conformation promoted by anesthetic binding near Val-136 in the wild-type channel. TASK-3 V136E, although resistant to TCE and isoflurane activation, itself retains near wild-type sensitivity to $\mathrm{m} 3$ GPCR inhibition (Fig. 8). This implies that the V136E mutation, relative to L122D, has fewer effects on TASK-3 gating and a potentially greater role in anesthetic binding. N133D, like L122D and similarly resistant to $\mathrm{m} 3 \mathrm{GPCR}$ inhibition (Fig. 8), may also have gating effects.

A Common Mechanism of Action and a Technical Advance. TASK-3 mutants resistant to activation by TCE are also resistant to activation by isoflurane. This was true for L122D, M159W, and V136E. Though not definitive, this is consistent with both agents activating TASK-3 through a common mechanism. Therefore, results determined with one agent likely apply to both pending verification. Our results are also a technical advance in the study of TASK-3 anesthetic pharmacology. Because volatile anesthetics volatilize, they are difficult to handle and to apply in a quantitative manner, particularly for prolonged periods. Halogenated alcohols, relative to volatile agents, have lower vapor pressures and increased aqueous solubility and can be handled in a more routine fashion with less concern for evaporative losses. This property enabled TBE studies on yeast growth over the time course of days and sets the stage for high-throughput and simultaneous screening of multiple TASK-3 and other tandem pore channel mutants.
Future Studies and Implications. Future studies might address the potency and efficacy of the brominated agents in activating other members of the tandem pore family. With their potency and ease of handling, the brominated compounds (TBE and $\mathrm{CBr}_{4}$ ) may be useful in crystallography studies or in developing a high-potency, nonvolatile photolabel for selective photolabeling of TASK-3 binding-site residues. In the end, a better understanding of TASK channel activation will guide rational design of potent and selective activators. TASK activators may have clinical utility as sedative hypnotics. TASK channels have a putative role in hypoxia sensing in the carotid body and in the pulmonary artery, and activators also may have utility in managing the adverse cardiovascular sequelae of sleep apnea and chronic hypoxia, and in treating pulmonary hypertension.

\section{Acknowledgments}

The authors thank Drs. Richard Gaber (Northwestern) and Dan Minor (UCSF) for supplying yeast reagents and for guidance in their use, and Dr. Dan Bolon (UMass) for helpful discussions on yeast selection and next generation sequencing. LyTASK cDNA was provided by Dr. Nick Franks (Imperial College). The authors also thank their MGH colleagues Drs. Brijesh Bhayana, Hua-Jun Feng, Stuart Forman, Youssef Jounaidi, Keith Miller, and Douglas Raines for helpful discussions.

\section{Authorship Contributions}

Participated in research design: Luethy, Boghosian, Cotten.

Conducted experiments: Luethy, Boghosian, Cotten.

Contributed new reagents or analytic tools: Luethy, Boghosian, Cotten.

Performed data analysis: Luethy, Cotten.

Wrote or contributed to the writing of the manuscript: Luethy, Boghosian, Srikantha, Cotten.

\section{References}

Andres-Enguix I, Caley A, Yustos R, Schumacher MA, Spanu PD, Dickinson R, Maze M, and Franks NP (2007) Determinants of the anesthetic sensitivity of two-pore domain acid-sensitive potassium channels: molecular cloning of an anestheticactivated potassium channel from Lymnaea stagnalis. J Biol Chem 282: 20977-20990.

Aryal P, Abd-Wahab F, Bucci G, Sansom MS, and Tucker SJ (2014) A hydrophobic barrier deep within the inner pore of the TWIK-1 K2P potassium channel. Nat Commun 5:4377.

Aryal P, Abd-Wahab F, Bucci G, Sansom MS, and Tucker SJ (2015) Influence of lipids on the hydrophobic barrier within the pore of the TWIK-1 K2P channel. Channels (Austin) 9:44-49.

Bagriantsev SN and Minor, JrDL (2013) Using yeast to study potassium channel function and interactions with small molecules. Methods Mol Biol 995:31-42.

Bertaccini EJ, Dickinson R, Trudell JR, and Franks NP (2014) Molecular modeling of a tandem two pore domain potassium channel reveals a putative binding site for general anesthetics. ACS Chem Neurosci 5:1246-1252.

Bertaccini EJ, Trudell JR, and Franks NP (2007) The common chemical motifs within anesthetic binding sites. Anesth Analg 104:318-324.

Brohawn SG, Campbell EB, and MacKinnon R (2014) Physical mechanism for gating and mechanosensitivity of the human TRAAK K+ channel. Nature 516:126-130.

Brohawn SG, del Mármol J, and MacKinnon R (2012) Crystal structure of the human K2P TRAAK, a lipid- and mechano-sensitive K+ ion channel. Science 335:436-441. Cadwell RC and Joyce GF (1994) Mutagenic PCR. PCR Methods Appl 3:S136-140. Chokshi RH, Larsen AT, Bhayana B, and Cotten JF (2015) Breathing stimulant compounds inhibit TASK-3 potassium channel function likely by binding at a common site in the channel pore. Mol Pharmacol 88:926-934.

Conway KE and Cotten JF (2012) Covalent modification of a volatile anesthetic regulatory site activates TASK-3 (KCNK9) tandem-pore potassium channels. Mol Pharmacol 81:393-400.

Dong YY, Pike AC, Mackenzie A, McClenaghan C, Aryal P, Dong L, Quigley A Grieben M, Goubin S, Mukhopadhyay S, et al. (2015) K2P channel gating mechanisms revealed by structures of TREK-2 and a complex with Prozac. Science $\mathbf{3 4 7}$ 1256-1259.

Firestone LL, Miller JC, and Miller KW (1986) Tables of physical and pharmacological properties of anesthetics, in Molecular and Cellular Mechanisms of Anesthetics (Roth SH and Miller KW eds) pp 455-470, Plenum Press, New York.

Franks NP (2008) General anaesthesia: from molecular targets to neuronal pathways of sleep and arousal. Nat Rev Neurosci 9:370-386.

Franks NP and Lieb WR (1988) Volatile general anaesthetics activate a novel neuronal K+ current. Nature 333:662-664. 
Gruss M, Bushell TJ, Bright DP, Lieb WR, Mathie A, and Franks NP (2004) Twopore-domain $\mathrm{K}+$ channels are a novel target for the anesthetic gases xenon, nitrous oxide, and cyclopropane. Mol Pharmacol 65:443-452.

Harinath S and Sikdar SK (2004) Trichloroethanol enhances the activity of recombinant human TREK-1 and TRAAK channels. Neuropharmacology 46 $750-760$.

Heurteaux C, Guy N, Laigle C, Blondeau N, Duprat F, Mazzuca M, Lang-Lazdunski L, Widmann C, Zanzouri M, Romey G, et al. (2004) TREK-1, a K+ channel involved in neuroprotection and general anesthesia. EMBO J 23:2684-2695.

Hönemann CW, Washington J, Hönemann MC, Nietgen GW, and Durieux ME (1998) Partition coefficients of volatile anesthetics in aqueous electrolyte solutions at various temperatures. Anesthesiology 89:1032-1035.

Kindler CH, Yost CS, and Gray AT (1999) Local anesthetic inhibition of baseline potassium channels with two pore domains in tandem. Anesthesiology 90: 1092-1102.

Krasowski MD, Finn SE, Ye Q, and Harrison NL (1998) Trichloroethanol modulation of recombinant GABAA, glycine and GABA rho 1 receptors. J Pharmacol Exp Ther 284:934-942.

Krasowski MD and Harrison NL (2000) The actions of ether, alcohol and alkane general anaesthetics on GABAA and glycine receptors and the effects of TM2 and TM3 mutations. Br J Pharmacol 129:731-743.

Lazarenko RM, Willcox SC, Shu S, Berg AP, Jevtovic-Todorovic V, Talley EM, Chen X, and Bayliss DA (2010) Motoneuronal TASK channels contribute to immobilizing effects of inhalational general anesthetics. $J$ Neurosci 30: 7691-7704.

Lolicato M, Riegelhaupt PM, Arrigoni C, Clark KA, and Minor, JrDL (2014) Transmembrane helix straightening and buckling underlies activation of mechanosensitive and thermosensitive K(2P) channels. Neuron 84:1198-1212.

Miller AN and Long SB (2012) Crystal structure of the human two-pore domain potassium channel K2P1. Science 335:432-436.

Nakamura RL and Gaber RF (1998) Studying ion channels using yeast genetics. Methods Enzymol 293:89-104.

Pang DS, Robledo CJ, Carr DR, Gent TC, Vyssotski AL, Caley A, Zecharia AY, Wisden W, Brickley SG, and Franks NP (2009) An unexpected role for TASK-3 potassium channels in network oscillations with implications for sleep mechanisms and anesthetic action. Proc Natl Acad Sci USA 106:17546-17551.
Parelkar NK, Silswal N, Jansen K, Vaughn J, Bryan, JrRM, and Andresen J (2010) 2,2,2trichloroethanol activates a nonclassical potassium channel in cerebrovascular smooth muscle and dilates the middle cerebral artery. J Pharmacol Exp Ther 332:803-810.

Patel AJ, Honoré E, Lesage F, Fink M, Romey G, and Lazdunski M (1999) Inhalational anesthetics activate two-pore-domain background $\mathrm{K}+$ channels. Nat Neurosci 2:422-426.

Pettersen EF, Goddard TD, Huang CC, Couch GS, Greenblatt DM, Meng EC, and Ferrin TE (2004) UCSF Chimera-a visualization system for exploratory research and analysis. J Comput Chem 25:1605-1612.

Poulsen H and Nissen P (2012) Structural biology. The inner workings of a dynamic duo. Science 335:416-417.

Putzke C, Wemhöner K, Sachse FB, Rinné S, Schlichthörl G, Li XT, Jaé L, Eckhardt I, Wischmeyer E, Wulf H, et al. (2007) The acid-sensitive potassium channel TASK1 in rat cardiac muscle. Cardiovasc Res 75:59-68.

Renigunta V, Yuan H, Zuzarte M, Rinné S, Koch A, Wischmeyer E, Schlichthörl G, Gao Y, Karschin A, Jacob R, et al. (2006) The retention factor P11 confers an endoplasmic reticulum-localization signal to the potassium channel TASK-1. Traffic 7:168-181.

Roth R, Gillespie D, Nonner W, and Eisenberg RE (2008) Bubbles, gating, and anesthetics in ion channels. Biophys J 94:4282-4298.

Sheppard DN, Carson MR, Ostedgaard LS, Denning GM, and Welsh MJ (1994) Expression of cystic fibrosis transmembrane conductance regulator in a model epithelium. Am J Physiol 266:L405-L413.

Talley EM and Bayliss DA (2002) Modulation of TASK-1 (Kcnk3) and TASK-3 (Kcnk9) potassium channels: volatile anesthetics and neurotransmitters share a molecular site of action. J Biol Chem 277:17733-17742.

Winegar BD and Yost CS (1998) Activation of single potassium channels in rat cerebellar granule cells by volatile anesthetics. Toxicol Lett 100-101:287-291.

Yost CS (2003) Update on tandem pore (2P) domain K+ channels. Curr Drug Targets 4:347-351.

Address correspondence to: Dr. Joseph F. Cotten, Massachusetts General Hospital, Department of Anesthesia, Critical Care, and Pain Medicine, 55 Fruit Street, GRB 444, Boston, MA 02114. E-mail: jcotten@mgh.harvard.edu 